\title{
Stabilization of volume gratings recorded in polyvinyl alcohol-acrylamide photopolymers with diffraction efficiencies higher than $\mathbf{9 0 \%}$
}

\author{
SERGI GALLEGO $\dagger$, CRISTIAN NEIPP†, \\ MANUEL ORTUNO $†$, AUGUSTO BELÉNDEZ† and \\ INMACULADA PASCUAL $\dagger$ \\ $\dagger$ Departamento Interuniversitario de Óptica, Universidad de Alicante, \\ Apartado 99, E-03080 Alicante, Spain; e-mail:pascual@ua.es \\ tDepartamento de Física, Ingeniería de Sistemas y Teoría de la Señal, \\ Universidad de Alicante, Apartado 99, E-03080 Alicante, Spain
}

(Received 16 April 2003; revision received 27 June 2003)

\begin{abstract}
Obtaining holographic volume gratings with high diffraction efficiencies that can be used under white light has been a serious problem for the polyvinyl alcohol-acrylamide-based photopolymers developed by other researchers. In this paper we propose to eliminate the residual monomer in order to stabilize the holographic gratings. The residual dye and residual monomer are the main problems in achieving high diffraction efficiencies stable under white light. In order to polymerize the residual monomer we illuminate the gratings with coherent green light and incoherent white light and we heat the grating at $80^{\circ} \mathrm{C}$ for different times. We also study the conservation of gratings dried in critical conditions of humidity and temperature. After stabilization the diffraction efficiencies achieved were clearly higher than $90 \%$.
\end{abstract}

\section{Introduction}

Photopolymers are a good holographic recording material because of their many attractive features [1]. Characteristics such as self-development, large dynamic range, good optical properties, thick emulsion layers (high angular selectivity) and relatively low cost make photopolymers one of the most promising materials for write-once read-many (WORM) holographic data storage applications $[2,3]$. Holograms need to be very thick in order to obtain a high information storage capacity, 1000 times greater than that of a CDROM, and random access time of only $10 \%$ of the latter [3]. Furthermore, this property allows us to record volume holographic gratings with high angular selectivity. Gratings can be used as enhanced edges [4] and in holographic interferometry [5]. Another application of holographic recording materials is the construction of holographic optical elements. For these applications, we need holographic gratings with a minimum transmission efficiency and maximum diffraction efficiency.

In order to obtain gratings recorded on poly(vinyl alcohol)PVA-acrylamide (AA) with maximum diffraction efficiency and minimum transmission efficiency at the Bragg angle, we need a good understanding of the behaviour of the photopolymer system. The mechanism that occurs in the recording process is complex but has been modelled successfully by many workers. This type of theoretical

Journal of Modern Optics ISSN 0950-0340 print/ISSN 1362-3044 online (C) 2003 Taylor \& Francis Ltd

http://www.tandf.co.uk/journals

DOI: $10.1080 / 09500340310001603168$ 
model is based on two main processes: monomer polymerization and monomer diffusion. This simplification is useful to characterize many aspects of the material (energy sensitivity, maximum diffraction efficiency attained, overmodulation, the presence of two peaks on the diffraction efficiency versus time graph, residual monomer, etc.) [6-12]. In this paper we use the model proposed by Neipp et al. [11] to explain the behaviour of the photopolymer. The applicability of this model to PVA - AA has been demonstrated in other studies [11].

In this paper we work with PVA-AA photopolymer to which we added $\mathrm{N}, \mathrm{N}$-methylene-bis-acrylamide (BMAA) as cross-linker. The behaviour of this type of photopolymer during the recording period is well known [13-17] and we can obtain volume holographic gratings with diffraction efficiencies higher than 95\%, taking into account the Fresnel losses. However, the stability of these gratings depends strongly on small variations in laboratory conditions (relative humidity and temperature). The diffraction efficiency is constant only under red light since, if we illuminate the hologram with white light, the residual dye and monomer react $[17,18]$. For this reason, refractive index modulation is lost and the diffraction efficiency decreases. In this study, our aim is to obtain diffraction gratings with maximum diffraction efficiency and minimum transmission efficiency at the Bragg angle that remain constant under white light, so that holographic optical elements may be made.

In other papers, the image recorded on photopolymers is stabilized by exposure to ultraviolet light, since in the photopolymers used there is a component that is sensitive at this wavelength. The function of this component is to catalyse the residual dye when the material is illuminated at this wavelength and then the hologram is stabilized [18]. However, our photopolymer does not have this component and it did not undergo tanning.

Other ways of stabilizing the photopolymer after the recording process with optimum proprieties at Bragg's angle are as follows. Firstly, we obtain overmodulated gratings. When the index modulation is higher than optimum, we use several methods in order to polymerize the residual monomer. This polymerization is the reason why the refractive index modulation decreases (by about 30\%). If during the recording process we reach a sufficiently high index modulation, when all the residual monomer polymerizes, the refractive index modulation will be that required to obtain maximum diffraction efficiency and minimum transmission efficiency at the Bragg angle. Moreover, if this volume grating is exposed to white light, all the parameters (thickness, absorption constant and refraction index modulation) remain constant. The methods used to eliminate the residual monomer and residual dye consist in placing the photopolymer under green light $(514 \mathrm{~nm})$, under white light (luminance of $1200 \mathrm{lux}$ ) and a heating process, since the acrylamide and bisacrylamide polymerize when the temperature is sufficiently high [19]. Finally, we analyse the conservation of gratings recorded under extreme conditions of temperature and humidity.

\section{Experimental set-up}

The photopolymerizable solution was prepared under red light by adding yellowish $\operatorname{eosin}(\mathrm{YE})$, as a dye, together with triethanolamine (TEA) to a solution of PVA(18-88 Fluka). The concentration of each of the components with water as solvent can be seen in table 1 . The resulting solution was deposited on a 
Table 1. Components of the photopolymerizable solution with cross-linker.

\begin{tabular}{ll}
\hline AA & $0.40 \mathrm{M}$ \\
BMAA & $0.04 \mathrm{M}$ \\
TEA & $0.20 \mathrm{M}$ \\
YE & $2.5 \times 10^{-4} \mathrm{M}$ \\
PVA & $7 \% \mathrm{w} / \mathrm{v}$ \\
\hline
\end{tabular}

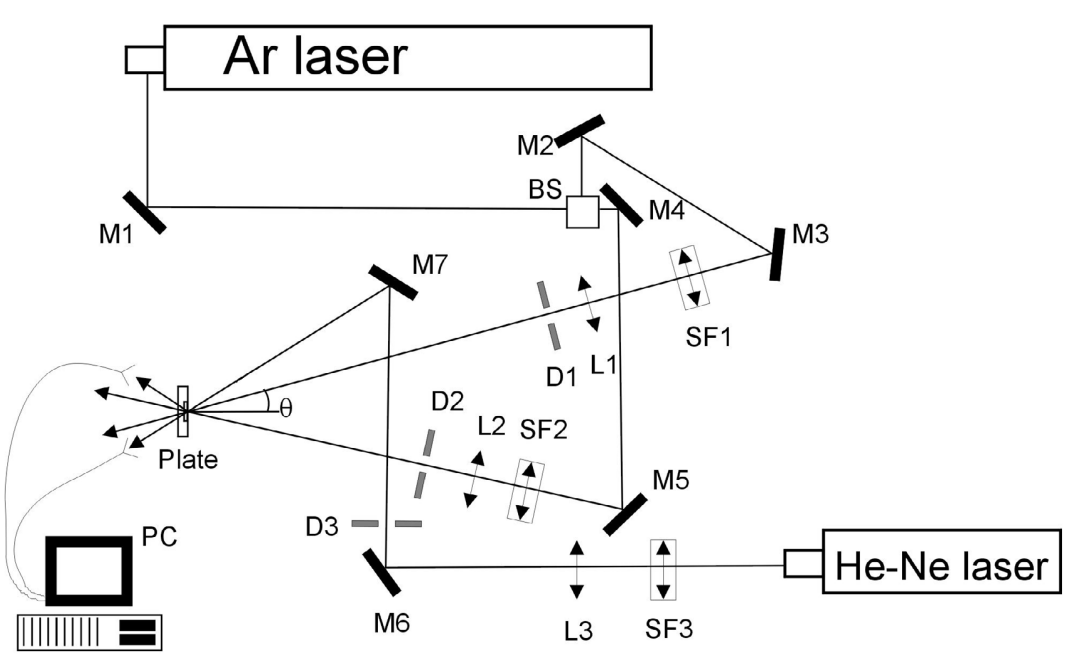

Figure 1. Experimental set-up: BS, beam splitter, Mi, mirrors; $\mathrm{SF} i$, spatial filters; $\mathrm{L} i$, lens; D $i$, diaphragm; PC, personal computer.

$20 \mathrm{~cm} \times 40 \mathrm{~cm}$ glass plate using an automatic depositor and adjusting the thickness of the film. The plate was dried for a period of $35-48 \mathrm{~h}$ in the dark and under normal laboratory conditions $\left(\mathrm{T}=22-23^{\circ} \mathrm{C}\right.$, relative humidity, $\left.50-60 \%\right)$. This type of photopolymer is very sensitive to laboratory conditions owing to the low solubility of BMAA in water; therefore, the drying time must be modified when there are slight variations in temperature or humidity. Once dried, the photopolymer was cut into plates measuring $6.5 \mathrm{~cm} \times 6.5 \mathrm{~cm}$ to be used in our experimental set-up, and the thickness, absorption constant and refractive index modulation were measured by optical methods [17].

To study the behaviour of the photopolymer as a holographic recording material, we obtained diffraction gratings using a holographic set-up. The experimental device is a typical holographic set-up [17], and it can seen in figure 1 . An argon laser at a wavelength of $514 \mathrm{~nm}$ was used to store diffraction gratings by means of continuous laser exposure. The laser beam was split into two secondary beams with an intensity ratio of $1: 1$. The diameter of these beams was increased to $1 \mathrm{~cm}$ with an expander, while spatial filtering was ensured. The object and reference beams were recombined at the sample at an angle of $16.8^{\circ}$ to the normal with an appropriate set of mirrors, and the spatial frequency obtained was 1125 lines $\mathrm{mm}^{-1}$. The working irradiance at $514 \mathrm{~nm}$ was $6 \mathrm{~mW} \mathrm{~cm}^{-2}$. The diffracted and transmitted intensity were monitored in real time with a $\mathrm{He}-\mathrm{Ne}$ laser positioned at the Bragg angle $\left(20.8^{\circ}\right)$ tuned to $633 \mathrm{~nm}$, where the material does not polymerize. In order to obtain the transmittance and diffraction efficiency as 
functions of the angle at reconstruction we placed the plates on a rotating stage. Transmittance and diffraction were calculated as the ratio of the transmitted and diffracted beam respectively to the incident power, and in order to take into account Fresnel losses the expression was multiplied by an appropriate factor.

\section{Results and discussion}

The idea expressed above of obtaining overmodulated gratings so that polymerization of the residual monomer results in the formation of gratings with the desired properties at the Bragg angle (low transmission efficiency and high diffraction efficiency) was very difficult to achieve with AA alone. In order to obtain overmodulation, the thickness or the refractive index modulation must be increased. Plates with AA as the only monomer are very unstable at high AA concentrations and noise gratings are formed with thick layers. Another possibility would be to include dimethylacrylamide so as to prevent spontaneous crystallization of the monomer; however, the gratings are not stable for very long [17]. In addition, we know that, if we add BMAA as cross-linker to the same initial monomer concentration, a higher index modulation (around 0.005 compared with 0.003 ) is obtained [12]. However, if we study the behaviour of volume holographic gratings at the Bragg angle predicted by Kogelnik's theory, it can be seen that, for the modulations achieved with the photopolymer used, exceptionally great thicknesses (around $90 \mu \mathrm{m}$ ) would be necessary for the curves to have the desired properties, that is for sufficient overmodulation to be obtained.

\subsection{Stabilization by incoherent light}

In this section, when the recording stage is completed, the grating is exposed to the white light (with a luminance of $1200 \mathrm{lux}$ ) to eliminate the residual monomer and stabilize the grating. The index modulation achieved after the recording stage decreases with time. Analysing the corresponding angular responses can see this.

Figure 2 shows the transmission efficiency as a function of exposure time for a $98 \mu \mathrm{m}$ plate thick. The experimental data were fitted to the first harmonic diffusion

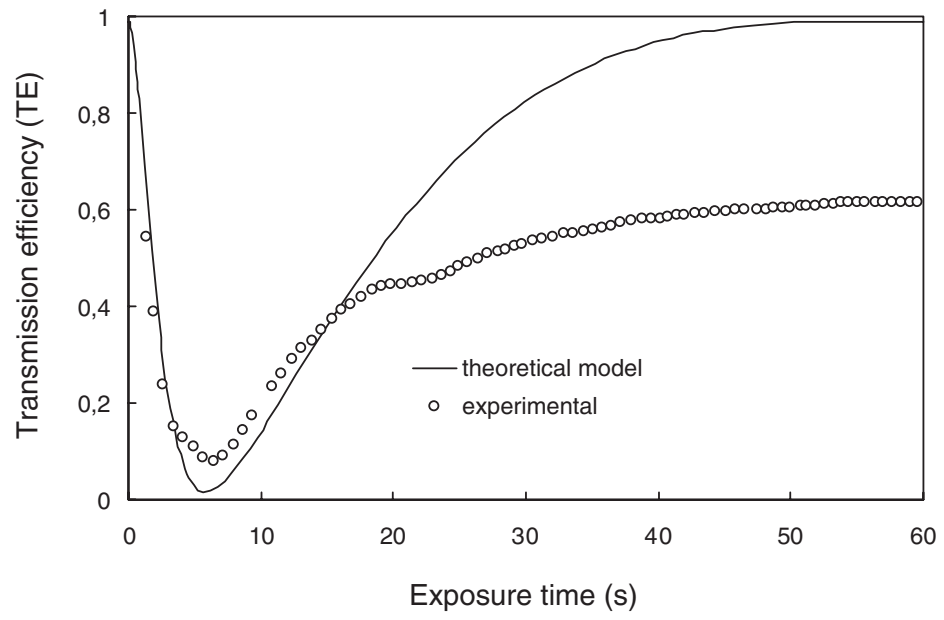

Figure 2. Transmission efficiency versus exposure time for a photopolymer $98 \mu \mathrm{m}$ thick. 


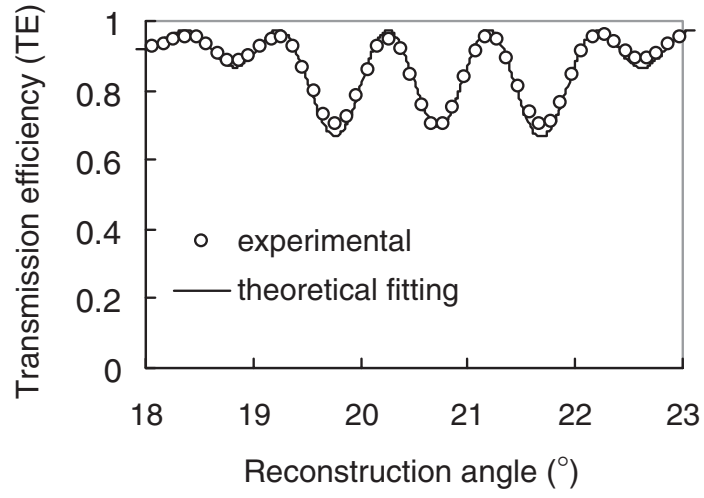

Figure 3. Transmission efficiency as a function of reconstruction angle immediately after exposure for a photopolymer $98 \mu \mathrm{m}$ thick $(\Delta n=0.0052)$.

model [11]. As can be seen in figure 2, a minimum transmission efficiency is obtained with a time of $7 \mathrm{~s}$, which means an energy sensitivity of $42 \mathrm{~mJ} \mathrm{~cm}^{-2}$ (the energy sensitivity is the minimun exposure to achieve maximun diffraction efficiency). After this moment, the index modulation continues to increase and the transmission efficiency also starts to increase as predicted by Kogelnik's theory when overmodulation occurs $[11,17]$. In this figure we can see that the theoretical fit differs from the experimental data at high exposures, and this effect has already been described in other studies [10]. The reason for this is that, in order to simplify the model, we did not take into account the increase in the time taken for diffusion to occur due to the presence of polymers in the exposed zones which prevent the monomer from diffusing to the non-exposed zones, as explained by Zhao and Mavolis [6].

The effects of overmodulation are confirmed if we analyse figure 3 which shows the fit of angular response based on Kogelnik's theory for unslanted pure phase gratings. In this way we obtain the values of thickness $d$, index modulation $\Delta n$ and absorption and scattering coefficient $\alpha(\Delta n=0.0052 \pm 0.0001, d=98 \pm 2 \mu \mathrm{m}$ and $\alpha=0.00034 \pm 0.00003 \mu \mathrm{m}^{-1}$ ).

Figure 4 shows the mean monomer and polymer concentrations relative to the initial monomer concentration in the photopolymer film during the recording stage according to our diffusion model. In figure 2 it can be seen that, when the experimental transmission efficiency is stabilized, its value is slightly higher than 0.6 . According to the theoretical diffusion model, this value is reached after $20 \mathrm{~s}$. In figure 4 , we can see that, at this moment (after $20 \mathrm{~s}$ ), there is slightly more than $30 \%$ residual monomer left. This monomer is found mainly in the dark zones, since in figure 2 it can be seen that, after the first $40 \mathrm{~s}$, the transmission efficiency is almost stabilized. In other words, in the bright zones there is almost no photopolymerizable solution left.

Figure 5 shows the temporal evolution of index modulation relative to the modulation obtained immediately after the grating is recorded. It can be seen that the grating is stabilized within the first $100 \mathrm{~h}$ of exposure and from this moment on the grating undergoes no further changes. The angular response of the grating, once it is stabilized, compared with the reading angle, is shown in figure 6 . As we can see, when index modulation is lost, the transmission efficiency in the 


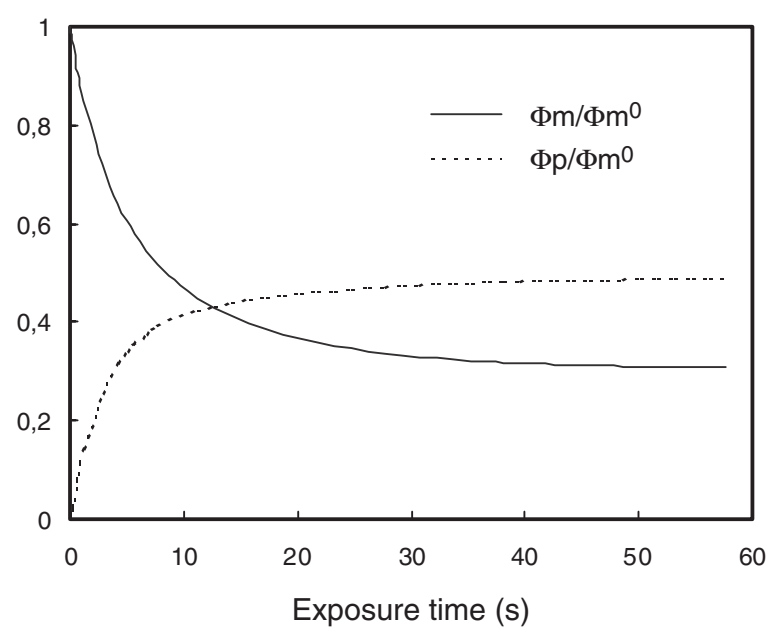

Figure 4. Monomer concentration $\Phi_{\mathrm{m}}$ divided by initial monomer concentration $\Phi_{m}^{0}$ and polymer concentration $\Phi_{\mathrm{p}}$ divided by initial monomer concentration as functions of exposure time for photopolymer $98 \mu \mathrm{m}$ thick.

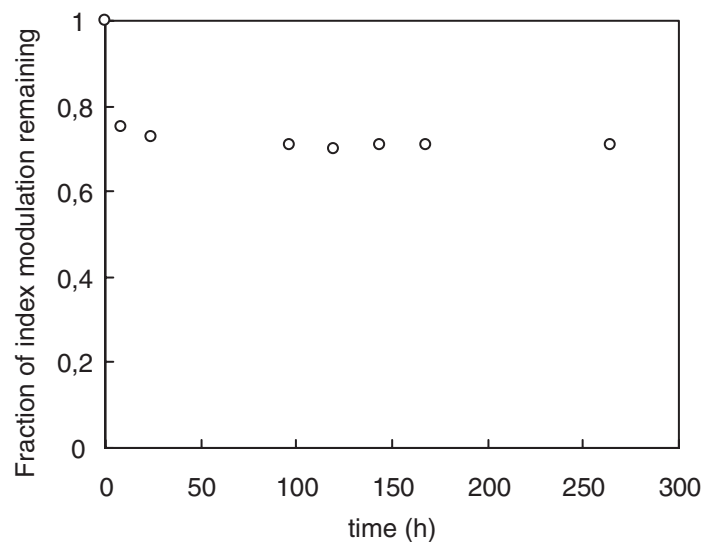

Figure 5. Refractive index modulation divided by the index modulation obtained immediately after recording as a function of time when the grating is kept under white ambient light (1200 lux).

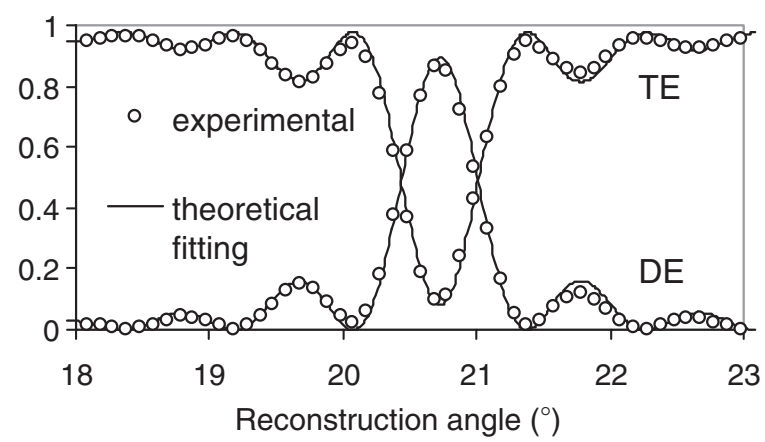

Figure 6. Transmission efficiency (TE) and diffraction efficiency (DE) as functions of reconstruction angle immediately after exposure, and $2000 \mathrm{~h}$ after the recording process. 
Bragg condition decreases to almost minimum values $(\Delta n=0.0037 \pm 0.0001$, $d=98 \pm 2 \mu \mathrm{m}$ and $\alpha=0.00025 \pm 0.000032 \mu \mathrm{m}^{-1}$ ). In other words, the diffraction efficiency increases until it reaches almost maximum values, thereby obtaining a diffraction grating with almost optimum results. In order to obtain a grating with optimum results (maximum diffraction efficiency and minimum transmission efficiency) for a thickness of $98 \mu \mathrm{m}$, the index modulation must take the value 0.0033 . For this to be possible, it would be necessary to stop recording the grating a little earlier so that there would be a greater amount of residual monomer present.

\subsection{Stabilization by coherent light}

We now use another plate of similar thickness $(96 \pm 2 \mu \mathrm{m})$ whose transmission efficiency versus time and angular response versus angle at reconstruction curves of the grating immediately after it is recorded are similar to the previous grating $\left(\Delta n=0.0052 \pm 0.0001, d=96 \pm 2 \mu \mathrm{m}\right.$ and $\left.\alpha=0.00027 \pm 0.00003 \mu \mathrm{m}^{-1}\right)$. However, in this case, once the diffraction grating is stored, it is exposed to an argon laser (a single beam) at an intensity of $30 \mathrm{~mW} \mathrm{~cm}^{-2}$ for $10 \mathrm{~min}$ in order to eliminate the residual monomer. In this time, all the dye in the plate is seen to have disappeared (other tests carried out with shorter exposure times indicate that there is still some residual monomer present). The angular response of the recorded grating was fitted after this treatment (the shape of the curve is like the curve represented in figure 6), and the parameters obtained from the fit are $\Delta n=0.0038 \pm 0.0001, d=95 \pm 2 \mu \mathrm{m}$ and $\alpha=0.0006 \pm 0.00003 \mu \mathrm{m}^{-1}$. This angular response remains constant after $2000 \mathrm{~h}$ and does not undergo any noticeable change. Moreover, as can be seen, although the index modulation of the grating decreases (from 0.0052 to 0.0038 ), when all the residual monomer has polymerized with the help of the laser, the behaviour at the Bragg angle is improved, since almost all the incident light is diffracted and only $5 \%$ is transmitted. Nevertheless, it should be pointed out that, if there were a slightly greater loss of refractive index modulation, or if we used a lower exposure or a somewhat thinner layer, the amount of light transmitted could be even less since, as can be deduced from the theoretical fit based on Kogelnik's theory, there is still a slight overmodulation in the angular response curve. In other words, a slightly lower index modulation (around 0.00325 instead of 0.0038 ) would be necessary to obtain minimum transmittance and maximum diffraction for a thickness of $95 \mu \mathrm{m}$.

\subsection{Stabilization by high exposure at a temperature of $80^{\circ} \mathrm{C}$}

As we explained above, in order to polymerize the residual monomer in the photosensitive film, we heated the overmodulated gratings at a temperature of $80^{\circ} \mathrm{C}$ for $60 \mathrm{~m}$. Figure 7 shows the evolution of the experimental data for transmission as a function of time for a plate $84 \mu \mathrm{m}$ thick together with the fit carried out on the basis of our model. Figure 8 shows the mean monomer and polymer concentrations that according to the model are present at each moment in the plate relative to the initial concentration of monomer. From figures 7 and 8 it can be deduced, as in section 3.1, that, when we stop recording the grating, around $30 \%$ of the monomer is still present. The angular response as a function of the angle at reconstruction of the grating at that particular moment may be seen in figure 9 , from the fit of which we obtain the thickness mentioned above. Transmittance 


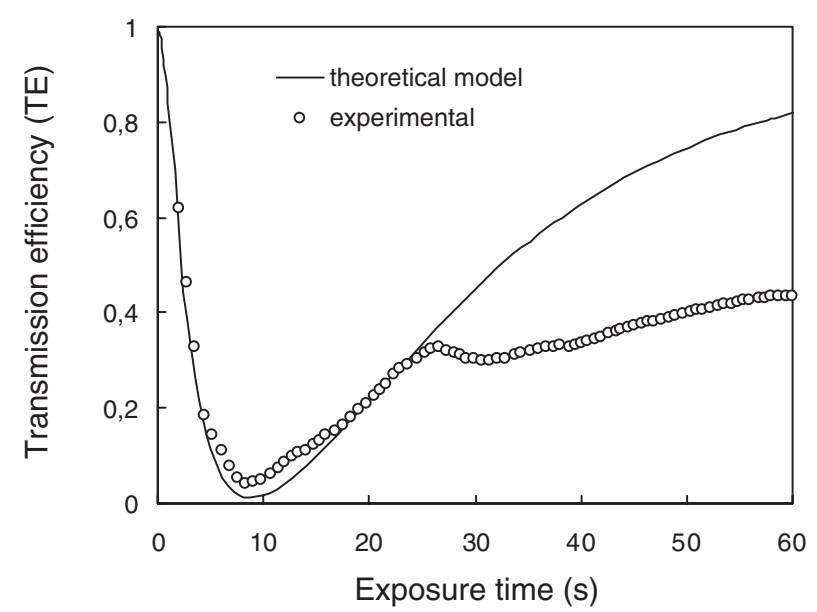

Figure 7. Transmission efficiency versus exposure time for a photopolymer $84 \mu \mathrm{m}$ thick.

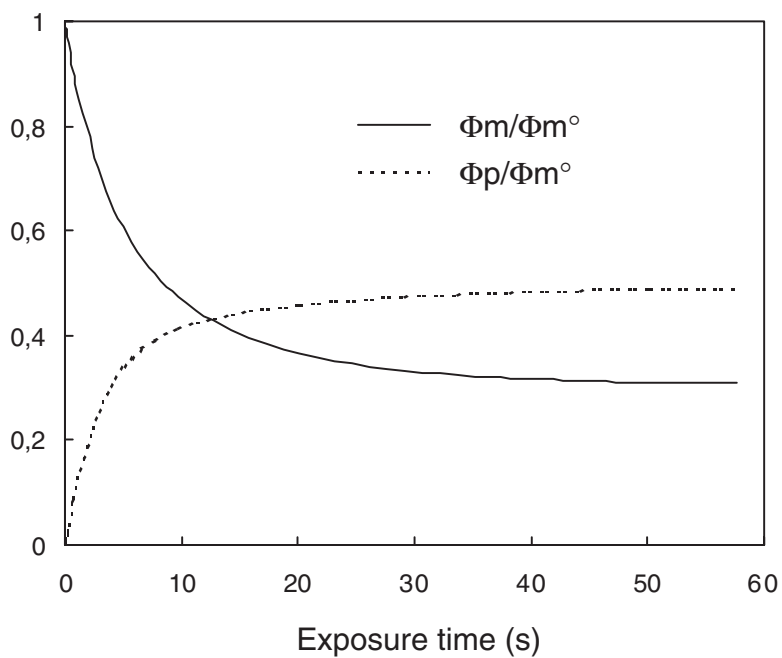

Figure 8. Monomer concentration $\Phi_{\mathrm{m}}$ divided by initial monomer concentration $\Phi_{m}^{0}$ and polymer concentration $\Phi_{\mathrm{p}}$ divided by initial monomer concentration as functions of exposure time for a photopolymer $84 \mu \mathrm{m}$ thick.

at the Bragg angle is approximately 0.4, which implies that the diffraction efficiency is around $60 \%$.

This grating was heated for $1 \mathrm{~h}$ at $80^{\circ} \mathrm{C}$ and then kept in the dark for $450 \mathrm{~h}$. Subsequently, the grating was exposed to the white light (ambient light) in order to determine whether there was still some unpolymerized monomer left. The evolution of the refractive index modulation compared with that obtained after recording the grating as a function of time may be seen in table 2. Variation in the thickness of the grating compared with the initial thickness can be seen in table 3 . As can be seen, in these tables, the index modulation decreases by about $20 \%$ in the first few hours and then remains constant up to $450 \mathrm{~h}$. At this moment the gratings are exposed to the light with the result that there is a further decrease in the index modulation. Meanwhile, the thickness of our grating decreases initially by a little 


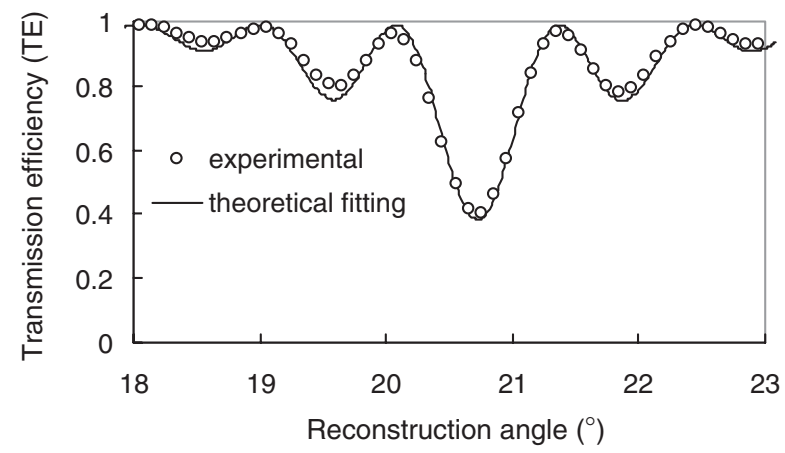

Figure 9. Transmission efficiency as a function of the reconstruction angle immediately after exposure for a photopolymer $84 \mu \mathrm{m}$ thick $(\Delta n=0.0050)$.

Table 2. Refractive index modulation divided by the index modulation obtained immediately after $(F)$ recording versus time.

\begin{tabular}{llllllll}
\hline$F \pm 0.03$ & 1 & 0.94 & 0.84 & 0.78 & 0.76 & 0.76 & 0.76 \\
Time (h)(stored in the dark) & 0 & 6 & 24 & 96 & 144 & 264 & 450 \\
$F \pm 0.03$ & 0.68 & 0.67 & 0.67 & 0.67 & 0.67 & 0.67 & 0.67 \\
Time (h)(stored in ambient light) & 464 & 480 & 520 & 604 & 696 & 2000 & 3000 \\
\hline
\end{tabular}

Table 3. Thickness divided by the thickness obtained immediately after recording $(D)$ versus time.

\begin{tabular}{llllllll}
\hline$D \pm 0.02$ & 1 & 0.89 & 0.97 & 0.97 & 1 & 1 & 1 \\
Time (h)(stored in the dark) & 0 & 6 & 24 & 96 & 144 & 264 & 450 \\
$D \pm 0.02$ & 1 & 1 & 1 & 1 & 1 & 1 & 1 \\
Time (h)(stored in ambient light) & 464 & 480 & 520 & 604 & 696 & 2000 & 3000 \\
\hline
\end{tabular}

more than $10 \%$ but then this loss in thickness is regained. These variations in thickness may be easily explained by the presence of PVA as binder. The function of PVA is to retain part of the water in the solution, thereby making the photosensitive film consistent. The amount of water retained depends on the PVA concentration and the humidity of the surroundings in which the film is kept [20]. For this reason, if we heat the photopolymer plate, part of the retained water evaporates and so the thickness decreases. However, when this process is over, the photopolymer returns to the laboratory conditions of temperature and relative humidity. The film will then absorb water from the atmosphere and so regain its initial thickness. These findings might lead us to think that all the residual monomer has polymerized but, when the recorded grating is exposed to the light (1200lux), $450 \mathrm{~h}$ after being recorded (table 2), we can see that it loses yet another $10 \%$. The parameters obtained by fitting the angular responses (transmission efficiency and diffraction efficiency) relative to the angle at reconstruction after $450 \mathrm{~h}$ (before exposure to the light) are $\Delta n=0.0040 \pm 0.0001, d=84 \pm 2 \mu \mathrm{m}$ and more than $95 \%$ of incident light was diffracted at the Bragg angle. The angular responses after $2000 \mathrm{~h}(450 \mathrm{~h}$ in the dark and $1550 \mathrm{~h}$ in the light) are shown in figure $10(\Delta n=0.0033 \pm 0.0001, d=84 \pm 2 \mu \mathrm{m})$, as we can see, the diffraction efficiency is around $95 \%$. 


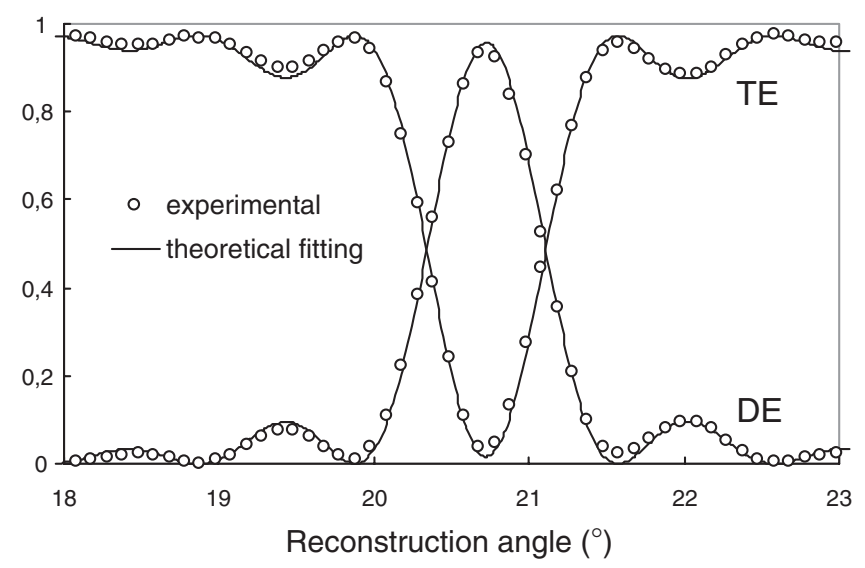

Figure 10. Transmission efficiency (TE) and diffraction efficiency (DE) as functions of reconstruction angle after $450 \mathrm{~h}$ in the dark and $1550 \mathrm{~h}$ under white light, $1200 \mathrm{lux}$ $(2000 \mathrm{~h}$ in total) for a photopolymer $84 \mu \mathrm{m}$ thick $(\Delta n=0.0034)$.

From analysis of the grating after $450 \mathrm{~h}$ (where there is still a slight overmodulation) and figure 10 it can be deduced that it is possible to store maximum diffraction efficiencies of over $95 \%$ at the Bragg angle, even though the time spent heating the plate $(1 \mathrm{~h})$ was not enough for all the residual monomer to polymerize, as can be seen when it is exposed to the light (table 2) after the first $450 \mathrm{~h}$.

This finding was to be expected if we consider the studies in which attempts were made to polymerize AA and BMAA by heating [19], when it is reported that $300 \mathrm{~min}$ are necessary to achieve maximum polymerization. For this reason, we used a plate of similar characteristics and heated it to $80^{\circ} \mathrm{C}$ for $200 \mathrm{~min}$. After this treatment, the refractive index modulation fell by $20 \%$ compared with the value that the grating had after the recording stage, and it did not vary when subsequently exposed to the light. However, we were faced with the drawback that this process resulted in a loss of $20 \%$ of the thickness which was not regained later. Therefore, it is necessary to control the heating so that the photopolymer film does not deteriorate irreversibly.

\subsection{Extreme laboratory conditions}

Other studies have pointed out the high sensitivity of PVA-AA based polymers to laboratory conditions and the influence that these have on the thickness, energy sensitivity, diffraction efficiency and stability of the plate. This latter characteristic, in particular, conditions our study since, if the temperature rises or the humidity decreases, our monomers crystallize very easily. Consequently, if these conditions change, the drying time must also change from $50 \mathrm{~h}$ at $20^{\circ} \mathrm{C}$ and $60 \%$ relative humidity to $20 \mathrm{~h}$ at $23^{\circ} \mathrm{C}$ and $44 \%$ relative humidity. However, if the conditions are very extreme as in the latter case (owing to the low humidity and high temperature), the plates may spontaneously crystallize after exposure, even immediately after they are cut, without the need for light. Therefore, it is interesting to note that the gratings that we recorded in these conditions are stable. Figure 11 shows the conservation of index modulation of a grating recorded in these conditions. During the first $60 \mathrm{~h}$ the grating was kept in the dark. In this period of time the grating lost $15 \%$ of the index modulation attained during 


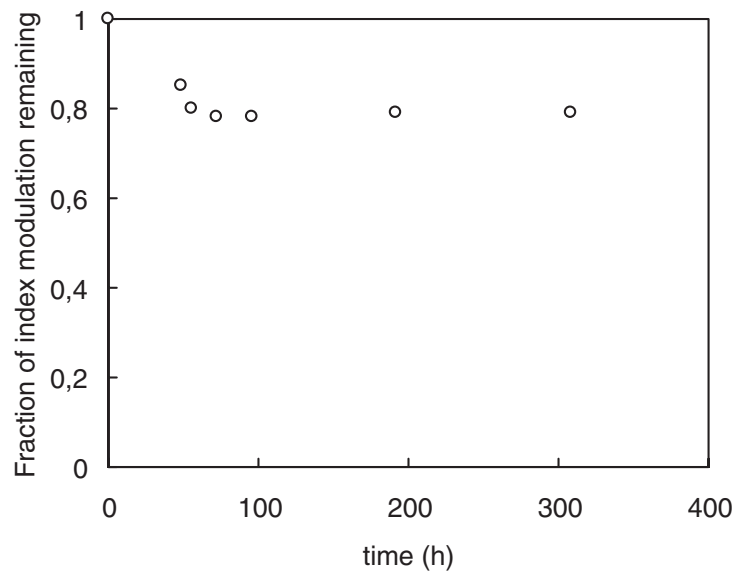

Figure 11. Refractive index modulation divided by the index modulation obtained immediately after recording as a function of time.

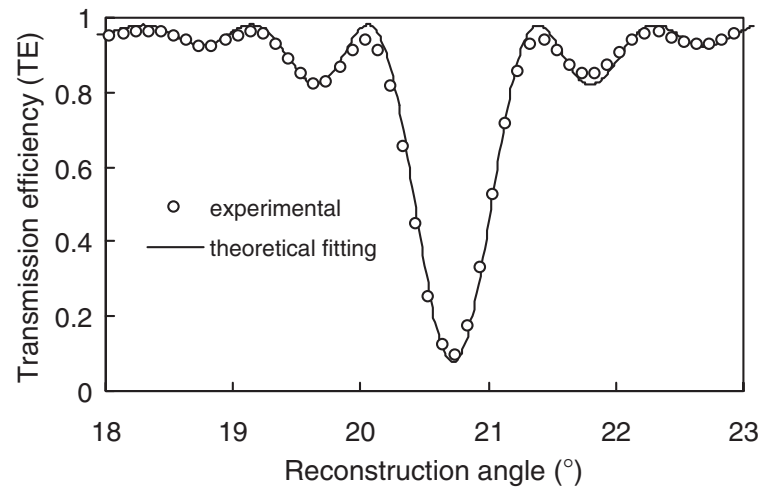

Figure 12. Transmission efficiency as a function of reconstruction angle immediately after exposure for a photopolymer $98 \mu \mathrm{m}$ thick $(\Delta n=0.0043)$.

recording. Moreover, there was considerable crystallization of AA and BMAA in the non-exposed zones, where the crystals could be seen, together with a notable loss of transparency. However, in the zones where the grating had formed, no crystals were to be seen, as in the previous cases in which the residual monomer was polymerized by the action of light or temperature, and so there was no loss of optical quality in our volume holographic grating. The grating was then exposed to the light in order to obtain an idea of the amount of residual monomer present. After approximately $40 \mathrm{~h}$ in these new conditions, $6 \%$ of the index modulation was lost before it became stabilized (this loss can be seen in more detail later in figure 13). This fact supports our idea that the mechanism by which index modulation is lost in the dark is not similar to the mechanism that occurs in photopolymers that do not contain a cross-linker but rather is mainly due to the effects commented on above.

Figure 12 shows the transmission efficiency relative to the angle at reconstruction immediately after the reconstruction stage $(\Delta n=0.0043 \pm 0.0001$, $d=99 \pm 2 \mu \mathrm{m}$ and $\alpha=0.00032 \pm 0.00003 \mu \mathrm{m}^{-1}$ ). When the angular response after the period spent in the dark was fitted, the follow parameters were 


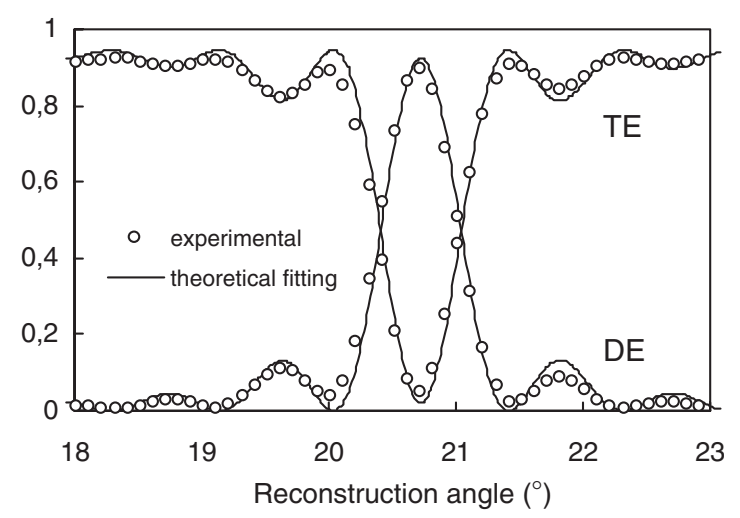

Figure 13. Transmission efficiency (TE) and diffraction efficiency (DE) as functions of reconstruction angle after $60 \mathrm{~h}$ in the dark and $240 \mathrm{~h}$ under white light, $1200 \mathrm{lux}$ (400 h in total) for a photopolymer $98 \mu \mathrm{m}$ thick $(\Delta n=0.0034)$.

obtained: $\Delta n=0.0037 \pm 0.0001, d=97 \pm 2 \mu \mathrm{m}$ and $\alpha=0.00020 \pm 0.00003 \mu \mathrm{m}^{-1}$. In figure $13(\Delta n=0.0034 \pm 0.0001, \quad d=97 \pm 2 \mu \mathrm{m} \quad$ and $\quad \alpha=0.00052 \pm$ $0.00003 \mu \mathrm{m}^{-1}$ ) the angular response (transmission efficiency and diffraction efficiency) after exposure to the light for $400 \mathrm{~h}$ (1200 lux) are shown when the index modulation had stabilized. We can see that this last angular response is almost the same as that desired at the Bragg angle, since the transmission efficiency has a value of less than $3 \%$ and the diffraction efficiency is over $91 \%$.

\section{Conclusion}

In this study we obtained gratings with optimum transmission and diffraction efficiencies, which were stable over time, at the Bragg angle, by means of different methods. Of the methods used, we believe that the most effective are those that consist in illuminating the grating with incoherent or coherent light, since heating the film causes problems due to the change in thickness of the hologram. Of the two types of illumination, coherent light cannot be considered more effective (a great deal of energy is needed to stabilize the plate) and so we recommend stabilizing the plate with ambient light because of its low cost. We also detected a 'spontaneous' loss of index modulation in the gratings which were dried and recorded under conditions of low humidity.

Finally, the following conclusion may be drawn from our study. If the correct laboratory temperature and relative humidity are chosen so that the diffusion time and reaction constant are always the same and depositing of the film is carefully controlled so that the desired thickness is obtained with a minimum error ( 1 or $2 \mu \mathrm{m}$ ), using the diffusion model, we can determine the time the photopolymer should be exposed so that after stabilization the desired transmission and diffraction efficiencies are obtained.

\section{Acknowledgments}

This work was supported by the Ministerio de Ciencia y Tecnología, Comisión Interministerial de Ciencia y Tecnología (Spain) under project 
MAT2000-1361-C04-04 and by the Oficina de Ciencia y Tecnología (Generalitat Valenciana, Spain) under project GV01-130.

\section{References}

[1] Lessard, R. A., and Manivannan, G., (editors), 1996, Selected Papers on Photopolymers (Bellingham, Washington: SPIE Optical Engineering Press), MS 114.

[2] Coufal, H. J., and Psaltis, D., 2000, Holographic Data Storage, edited by G.T. Sincerbox (New Cork: Springer), p.181.

[3] Van Heerden, P. J., 1963, Appl. Optics, 2, 393.

[4] Márquez, A., Neipp, C., Gallego, S., Ortuño, M., Pascual, I., and Beléndez, A., 2003, Optics Lett. (to be published).

[5] Martin, S., Leclere, P. E. L. G., Toal, V., and Lion, Y. F., 1997, Appl. Optics, 36, 5757-5768.

[6] Zhao, G., and Mourolis, P., 1994, J. mod. Optics, 41, 1929.

[7] Aubrecht, I., Miler, M., and Koudela, I.,1998, J. mod. Optics, 45, 1465.

[8] Kwon, J. H., Hwang, H. C., and Woo, K. C., 1999, J. opt. Soc. Am. B, 16, 1651.

[9] Piazolla, S., and Jenkins, B. J., 2000, J. opt. Soc. Am. B, 17, 1147.

[10] Sheridan, J. T., Downey, M., and O’Neill, F. T., 2001, J. Optics A, 3, 477

[11] Neipp, C., Gallego, S., Ortuño, M., Márquez, A., Álvarez, M., Beléndez, A., and Pascual, I., 2003, J. opt. Soc. Am. B (to be published).

[12] Martin, S., Leclere, P. E. L. G., Toal, V., and Lion, Y. F., 1994, Opt. Enging, 33, 3942.

[13] Weiss, V., Millul, E., and Friesem, A., 1996, Holographic Materials II, Proceedings of SPIE, Vol. 2688 (Bellingham, Washington: SPIE).

[14] Blaya, S., Carretero, L., Mallavia, R., Fimia, A., Ulibarrena, M., and Levy, D., 1998, Appl. Optics, 37, 7604.

[15] García, C., Fimia, A., and Pascual, I., 1999, Appl. Optics, 38, 5548.

[16] García, C., Fimia, A., and Pascual, I., 2001, Appl. Phys. 72, 311.

[17] Gallego, S., Ortuño, M., Neipp, C., García, C., Beléndez, A., and Pascual, I., 2003, Optics Express, 11, 181.

[18] Jenney, J. A., 1970, J. opt. Soc. Am., 60, 1155.

[19] Donovan, M. S., Sanford, T. A., Lowe, A. B., Sumerlin, B. S., Mitsukami, Y., and McCormick, C. L., 2002, Macromolecules, 35, 4570.

[20] Ortuño, M., Gallego, S., García, C., Neipp, C., Beléndez, A., and Pascual, I., 2003, Appl. Phy. B (to be published). 


\section{AUTHOR QUERIES}

JOURNAL ID: TMOP-102020

QUERY

NUMBER

1
QUERY

Further details? 